\title{
Te Acercamos El Mundo Por Que Tus Sueños Viajan Con Nosotros. Estudio De Caso: Implementación De Una Agencia De Viajes Y Operadora De Turismo En La Ciudad De Riobamba Provincia De Chimborazo
}

\author{
Rita Sulema Lara Vásconez \\ Flor Quinchuela \\ Jenry Ernesto López Romero \\ Docentes investigadores Escuela de Ingeniería en Ecoturismo/Escuela \\ Superior Politécnica de Chimborazo/Ecuador
}

doi: 10.19044/esj.2016.v12n17p349 URL:http://dx.doi.org/10.19044/esj.2016.v12n17p349

\begin{abstract}
The study of the facility permitted to determine that the implementation of the travel agency and the operator of tourism Sumak TourismRio in the city of Riobamba, in Chimborazo Province is possible or viable. Techiques of documentary and local investigations were used. In order to accomplish this investigation, a fundamental point of reference, the commercial viability of the project, was taken, which is based on the study of the market. This study was done considering the analysis of the demand. For this an instrument of investigation that in this case was the inquest guide was applied, which permitted the direct collection of information. On the other hand an analysis of the competition considering the local investigation was made considering the tourism organizations (travel agencies and operators of tourism) of the city of Riobamba which offer tourist products of similar characters. Furthermore the projection of the future demand and future competition, using the method of combined increment whose mathematatic method is: $\mathrm{Cn}=\mathrm{Co}(1+\mathrm{i})$. Also the unsatisfacatory existence, that is the confrontation of these factors considering un a difference. It is valid review the importance of the analysis of the demand, since it contributes to the definition of the description of the tourist. Considering all the information collected, the following was defined: the universe of the study shows different requirements in reference to tourist packets that the company offers, which permits economic benefits and by this means activate the economy of the city. Finally, it was shown that the sale of tourist packets Will permit the capitalization of the organization, producing a positive financial profit, since the investment produced will be recuperated in the first year of operation,
\end{abstract}


producing an internal profit of 1,493\%, an actual net amount of $\$ 2,625610.27$ (dollars) and a cost benefit of $\$ 104.19$.

Keywords: Travel agency, tourist operator, tourist packets factibility, viability, financial profits, demand, competition, Riobamba

\section{Resumen}

El estudio de factibilidad permitió determinar que la implementación de la agencia de viajes y operadora de turismo Sumak TourismRio en la ciudad de Riobamba, provincia de Chimborazo es factible o viable. Se utilizó técnicas de investigación documental y de campo. Para realizar esta investigación se tomó como eje fundamental la viabilidad comercial del proyecto la misma que se basa en el estudio de mercado. Dicho estudio de mercado se lo hizo en base al análisis de la demanda, para el mismo se aplicó un instrumento de investigación que este caso fue la guía de encuesta que permitió la recolección de información directa. Por otro lado se realizó el análisis de la competencia en base a la investigación de campo que consideró a las empresas turísticas (Agencias de viajes y operadoras de turismo) de la ciudad de Riobamba que ofrecen productos turísticos de similares características. Además se efectuó la proyección de la demanda futura y competencia futura utilizando el método de incremento compuesto cuyo modelo matemático es: $\mathbf{C n}=\mathbf{C o}(\mathbf{1}+\mathbf{i}){ }^{\mathbf{n}}$. También se estableció la demanda insatisfecha existente, es decir la confrontación de las mismas en base a una diferencia. Es válido recalcar la importancia del análisis de la demanda ya que contribuye a definir el perfil de turista. En base a la información recogida se definió lo siguiente: El universo de estudio muestra distintos requerimientos en cuanto a paquetes turísticos que ofrece la compañía lo que permitirá lograr beneficios económicos y de esa forma activar la economía de la ciudad. Finalmente se evidencio que la venta de paquetes turísticos permitirá la capitalización de la empresa generando una rentabilidad positiva, pues la inversión realizada se recuperará en el primer año de operación proporcionando una tasa interna de retorno del 1493\%, un valor actual neto de \$ \$2.625.610,27 dólares y una relación beneficio costo de \$104,19 dólares.

Palabras claves: Agencia de viajes, operadora de turismo, factibilidad, viabilidad, rentabilidad, demanda, competencia, Riobamba

\section{Introducción}

El turismo se ha convertido en un fenómeno mundial que genera, una fuerza económica muy importante. Según la Organización Mundial del Turismo (OMT) en el año 2005 los países de América del Sur habrían 
recibido alrededor de 18 millones de turistas internacionales, lo que representa un crecimiento del 12,7\% en ingreso de divisas. Para el año 2020 la OMT proyecta 1600 millones de llegadas de turistas internacionales y un volumen total de ingresos de 2 billones de dólares, se estima que el turismo interno supera en unas 10 veces el volumen mundial del turismo internacional por lo que el turismo sigue siendo uno de los principales puntales en el desarrollo. (OMT, 2010).

El turismo en el Ecuador es reconocido como uno de los sectores de mayor dinamismo, con una alta capacidad en la generación de empleos y divisas (SENPLADES, 2013), es por eso que el turismo es uno de los sectores priorizados para el cambio de la matriz productiva (MCPEyC, 2014).

El desarrollo del turismo ha servido de motor para impulsar a otros sectores de la economía nacional, uno de estos sectores es el conformado por los agentes de viajes y operadoras turísticas, ambos muy vinculados al turismo por la función que desempeñan al servir de nexo para facilitar el acceso de turistas al país. (MINTUR, 2013).

Es así que en el país están registradas 714 empresas, el 62,5\% corresponde a agencias de viajes internacionales, el 31,5\% a operadoras de turismo y el 6\% a agencias mayoristas. La mayor concentración de empresas de turismo se da en Quito (50,4\%); de estas, el 23\% son operadoras de servicios, sobre todo porque es en esta ciudad donde están concentradas las operaciones de servicios hacia las diferentes regiones del país. Este hecho obedece también a que Quito ofrece una mejor infraestructura de recepción, conectividad y comunicaciones, aspecto que han favorecido las iniciativas de desarrollo de productos, servicios y mercados en el campo del turismo. (MINTUR, 2013).

Según el Barómetro Turístico 2011 “Nuestro país posee una amplia oferta de recursos turísticos, tanto naturales como culturales, por lo cual el Estado, en sus diversos niveles debe asumir en forma prioritaria un rol activo de coordinación y promoción de actividades del sector turístico y comprometer el apoyo a las acciones que la sociedad realiza con el fin de potencializar el sector”.

Existen cuatro regiones distintas, el llano tropical de la Costa, la cuenca del Amazonas, las Islas Galápagos y los andes montañosos. La provincia de Chimborazo se encuentra en el centro de los andes del Ecuador, su capital es Riobamba, se asienta en una amplia meseta, desde la que se divisan cumbres entre los que se destacan el nevado Chimborazo, Carihuairazo, Tungurahua y Altar. (MINTUR, 2013).

Riobamba tiene su importancia por ser lugar de tránsito obligado entre distintas regiones del país. Cuenta con interesantes parques, museos y monumentos, además de la riqueza cultural que es común en toda la 
provincia, por ser la que alberga el grupo más numeroso de población indígena. Ello se refleja en múltiples manifestaciones de la cultura popular. (BARÓMETRO TURÍSTICO 2011).

En la provincia se encuentran varios atractivos de tipo natural y cultural, entre ellos, se destacan Guano, conocido por sus alfombras artesanales de lana, Punín, donde se han encontrado restos humanos con una antigüedad superior a los 1000 años; Cajabamba, asentamiento de la primitiva Riobamba y lugar donde se fundó la primera ciudad de Santiago de Quito; Balbanera, la primera iglesia construida por los españoles en suelo ecuatoriano; la laguna de Colta donde crece totora, entre otras. (MINTUR, 2013).

El principal atractivo de la provincia es el nevado Chimborazo ubicado en la Reserva de Producción Faunística del mismo nombre. El Chimborazo, con sus 6310 metros de altura, se ha convertido en un lugar obligado de visita en Ecuador para aquellos turistas amantes del montañismo. En Riobamba se encuentran importantes andinistas del país, que operan y guían al turista hacia las cumbres de este nevado o del Altar que es un volcán ya extinguido y el Sangay es uno de los volcanes más activos del mundo, siendo frecuentes las erupciones de rocas incandescentes. (MINTUR, 2013).

Una de las alternativas, que de hecho será el motivo de este estudio, son las agencias operadoras, que han experimentado un considerable índice de crecimiento en lo que se refiere a número y operación, reflejando un mercado que se lo puede considerar prometedor, pero que por estar sujeto a una serie de factores que pueden afectar la inversión, es necesario realizar estudios que garanticen la factibilidad para la implementación de una empresa.

La presente investigación permitirá determinar la factibilidad para la implementación de una operadora de turismo y agencia de viajes que promueva el desarrollo turístico, a través de la operación de los distintos paquetes que se ofrecerán, generando beneficios económicos para la empresa y a su vez para los miembros de las zonas con los que se opere el producto, procurando que la población local mejore su calidad de vida bajo criterios de sostenibilidad.

\section{Método}

Para el presente trabajo se aplicaron los métodos y técnicas de la investigación de campo y documental.

\section{Análisis de la Demanda}

El servicio que se ofrecerá será dirigido a la población de la ciudad de Riobamba y de Guayaquil cuyas edades están comprendidas entre los 15- 
65 años que pertenecen a la clase media alta. El universo de estudio estuvo conformado los potenciales clientes que provienen de la localidad de Riobamba que en total son 51631 personas; mientras que los que proceden de la ciudad de Guayaquil son 553417 individuos de acuerdo al segmento de mercado (INEC, 2010). Luego se estableció la muestra la misma que fue calculada con un error del $5 \%$ y con un valor de confianza igual a 1.96, con una probabilidad de ocurrencia y no ocurrencia de 0.5 , obteniendo como resultado 381 personas para la ciudad de Riobamba, mientras que para la ciudad de Guayaquil se obtuvo 384 individuos a ser encuestados.

\section{Instrumento de Investigación}

El instrumento utilizado para recabar información fue la guía de encuesta que se encuentra en el anexo 1 para clientes de la ciudad de Riobamba y 2 aplicado a clientes provenientes de Guayaquil.

\section{Técnica}

La técnica empleada fue la encuesta que permitió la recolección de la información en forma directa.

\section{Análisis de la Competencia}

Para definir la competencia se consideró a las empresas turísticas (Agencias de viajes y operadoras de turismo) de la ciudad de Riobamba que ofrecen productos turísticos de similares características.

\section{Proyección de la Demanda Potencial y Competencia}

En base a la demanda y competencia actual se hicieron las proyecciones respectivas, utilizando el método de incremento compuesto cuyo modelo matemático es: $\mathbf{C n}=\mathbf{C o}(\mathbf{1}+\mathbf{i})^{\mathbf{n}}$

\section{Determinación de la Demanda Insatisfecha} diferencia.

Se confrontó la demanda y competencia futura en base a una 


\section{Resultados}

\section{Análisis de la Demanda}

\section{Perfil de los Potenciales Clientes de la Ciudad de Riobamba}

\section{Edad}

Cuadro 1. Edad de los potenciales clientes de la ciudad de Riobamba

\begin{tabular}{|c|c|c|c|c|}
\hline Edad & $\begin{array}{c}\text { Frecuencia } \\
\text { absoluta }\end{array}$ & $\begin{array}{c}\text { Frecuencia } \\
\text { acumulada }\end{array}$ & $\begin{array}{c}\text { Frecuencia } \\
\text { relativa }\end{array}$ & $\begin{array}{c}\text { Frecuencia } \\
\text { relativa } \\
\text { acumulada }\end{array}$ \\
\hline De 15 a 19 años & 48 & 48 & $12,5 \%$ & $12,5 \%$ \\
\hline De 20 a 24 años & 29 & 29 & $7,5 \%$ & $20 \%$ \\
\hline De 25 a 29 años & 86 & 86 & $22,5 \%$ & $42,5 \%$ \\
\hline De 30 a 34 años & 67 & 67 & $17,5 \%$ & $60 \%$ \\
\hline De 35 a 39 años & 38 & 38 & $10 \%$ & $70 \%$ \\
\hline De 40 a 44 años & 19 & 19 & $5 \%$ & $75 \%$ \\
\hline De 45 a 49 años & 10 & 10 & $2,5 \%$ & $77,5 \%$ \\
\hline De 50 a 54 años & 29 & 29 & $7,5 \%$ & $85 \%$ \\
\hline De 55 a 59 años & 38 & 38 & $10 \%$ & $95 \%$ \\
\hline De 60 a 64 años & 19 & 19 & $5 \%$ & $100 \%$ \\
\hline
\end{tabular}

El 22,5\% del universo de estudio tienen edades entre los 25 a 29 años, el 17,5\% tienen entre 30 y 34 años de edad y el 12,5\% se hallan entre los 15 y 19 años.

\section{Género}

Cuadro 2. Género de los potenciales clientes de la ciudad de Riobamba

\begin{tabular}{|c|c|c|c|c|}
\hline Género & $\begin{array}{c}\text { Frecuencia } \\
\text { absoluta }\end{array}$ & $\begin{array}{c}\text { Frecuencia } \\
\text { acumulada }\end{array}$ & $\begin{array}{c}\text { Frecuencia } \\
\text { relativa }\end{array}$ & $\begin{array}{c}\text { Frecuencia } \\
\text { relativa } \\
\text { acumulada }\end{array}$ \\
\hline Masculino & 42 & 42 & $35 \%$ & $35 \%$ \\
\hline Femenino & 77 & 119 & $65 \%$ & $100 \%$ \\
\hline
\end{tabular}

El 65\% de los potenciales clientes son del género femenino y el restante del género masculino.

\section{Nivel de instrucción}

Cuadro 3. Nivel de instrucción de los potenciales clientes de la ciudad de Riobamba

\begin{tabular}{|c|c|c|c|c|}
\hline Nivel de instrucción & $\begin{array}{c}\text { Frecuencia } \\
\text { absoluta }\end{array}$ & $\begin{array}{c}\text { Frecuencia } \\
\text { acumulada }\end{array}$ & $\begin{array}{c}\text { Frecuencia } \\
\text { relativa }\end{array}$ & $\begin{array}{c}\text { Frecuencia } \\
\text { relativa } \\
\text { acumulada }\end{array}$ \\
\hline Primaria & 48 & 48 & $12,5 \%$ & $12,5 \%$ \\
\hline Secundaria & 143 & 191 & $37,5 \%$ & $50 \%$ \\
\hline Superior & 162 & 352 & $42,5 \%$ & $93 \%$ \\
\hline Postgrado & 29 & 381 & $7,5 \%$ & $100 \%$ \\
\hline
\end{tabular}


El 42,5\% del universo de estudio tienen un nivel de instrucción superior, por otro lado el $37,5 \%$ han terminado la secundaria, mientras que el $12.5 \%$ solo culminaron la primaria.

\section{Procedencia}

Cuadro 4. Procedencia de los potenciales clientes de la ciudad de Riobamba

\begin{tabular}{|c|c|c|c|c|}
\hline Procedencia & $\begin{array}{c}\text { Frecuencia } \\
\text { absoluta }\end{array}$ & $\begin{array}{c}\text { Frecuencia } \\
\text { acumulada }\end{array}$ & $\begin{array}{c}\text { Frecuencia } \\
\text { relativa }\end{array}$ & $\begin{array}{c}\text { Frecuencia relativa } \\
\text { acumulada }\end{array}$ \\
\hline Riobamba & 276 & 276 & $72,5 \%$ & $72,5 \%$ \\
\hline Ambato & 19 & 295 & $5 \%$ & $77,5 \%$ \\
\hline Guayaquil & 19 & 314 & $5 \%$ & $82,5 \%$ \\
\hline Alausí & 10 & 324 & $2,5 \%$ & $85 \%$ \\
\hline Cevallos & 10 & 333 & $2,5 \%$ & $87,5 \%$ \\
\hline Galápagos & 10 & 343 & $2,5 \%$ & $90 \%$ \\
\hline Macas & 10 & 352 & $2,5 \%$ & $92,5 \%$ \\
\hline Guamote & 10 & 362 & $2,5 \%$ & $95 \%$ \\
\hline Machala & 10 & 371 & $2,5 \%$ & $97,5 \%$ \\
\hline Bucay & 10 & 381 & $2,5 \%$ & $100 \%$ \\
\hline
\end{tabular}

El 72,5\% de los potenciales clientes son de la ciudad de Riobamba, el $5 \%$ provienen de la ciudad de Ambato, un porcentaje similar proceden de Guayaquil y un 2,5\% vienen de Alausí.

\section{Ocupación}

Cuadro 5. Ocupación de los potenciales clientes de la ciudad de Riobamba

\begin{tabular}{|c|c|c|c|c|}
\hline Ocupación & $\begin{array}{c}\text { Frecuencia } \\
\text { absoluta }\end{array}$ & $\begin{array}{c}\text { Frecuencia } \\
\text { acumulada }\end{array}$ & $\begin{array}{c}\text { Frecuencia } \\
\text { relativa }\end{array}$ & $\begin{array}{c}\text { Frecuencia } \\
\text { relativa } \\
\text { acumulada }\end{array}$ \\
\hline Empleado público & 114 & 114 & $30 \%$ & $30 \%$ \\
\hline Empleado privado & 95 & 210 & $25 \%$ & $55 \%$ \\
\hline Estudiante & 76 & 286 & $20 \%$ & $75 \%$ \\
\hline Jubilado & 19 & 305 & $5 \%$ & $80 \%$ \\
\hline Ama de casa & 76 & 381 & $20 \%$ & $100 \%$ \\
\hline
\end{tabular}

El 30\% del universo de estudio son empleados del sector público, por otro lado el $25 \%$ laboran en el sector privado, mientras que el $20 \%$ son estudiantes. 


\section{Tipo de empresa a crearse}

Cuadro 6. Tipo de empresa a crearse según la preferencia de la población de Riobamba

\begin{tabular}{|c|c|c|c|c|}
\hline Tipo de empresa a crearse & $\begin{array}{c}\text { Frecuencia } \\
\text { absoluta }\end{array}$ & $\begin{array}{c}\text { Frecuencia } \\
\text { acumulada }\end{array}$ & $\begin{array}{c}\text { Frecuencia } \\
\text { relativa }\end{array}$ & $\begin{array}{c}\text { Frecuencia } \\
\text { relativa } \\
\text { acumulada }\end{array}$ \\
\hline $\begin{array}{c}\text { Operadora de turismo y agencia } \\
\text { de viajes }\end{array}$ & 267 & 267 & $70 \%$ & $70 \%$ \\
\hline Establecimiento hotelero & 67 & 333 & $18 \%$ & $87,5 \%$ \\
\hline $\begin{array}{c}\text { Establecimiento de comidas y } \\
\text { bebidas }\end{array}$ & 48 & 381 & $13 \%$ & $100 \%$ \\
\hline
\end{tabular}

El 70\% de los potenciales clientes prefieren que se implemente una operadora de turismo y agencia de viajes, el 17,5\% le gustaría que se cree un establecimiento hotelero y el restante está de acuerdo que se establezca un establecimiento de comidas y bebidas.

\section{Forma de viaje}

Cuadro 7. Forma de viajar de los potenciales clientes de la ciudad de Riobamba

\begin{tabular}{|c|c|c|c|c|}
\hline Forma de viaje & $\begin{array}{c}\text { Frecuencia } \\
\text { absoluta }\end{array}$ & $\begin{array}{c}\text { Frecuencia } \\
\text { acumulada }\end{array}$ & $\begin{array}{c}\text { Frecuencia } \\
\text { relativa }\end{array}$ & $\begin{array}{c}\text { Frecuencia } \\
\text { relativa } \\
\text { acumulada }\end{array}$ \\
\hline Sólo & 57 & 57 & $15 \%$ & $15 \%$ \\
\hline Pareja & 48 & 105 & $13 \%$ & $28 \%$ \\
\hline Familia & 181 & 286 & $48 \%$ & $75 \%$ \\
\hline Amigos & 67 & 352 & $18 \%$ & $93 \%$ \\
\hline Compañeros & 29 & 381 & $8 \%$ & $100 \%$ \\
\hline
\end{tabular}

El 48\% de los potenciales clientes prefieren viajar en familia, el 18\% viajan con amigos, y por su parte el $15 \%$ viajan solos.

\section{Frecuencia de viaje}

Cuadro 8. Frecuencia con la que los potenciales clientes de la ciudad de Riobamba viajan

\begin{tabular}{|c|c|c|c|c|}
\hline $\begin{array}{c}\text { Frecuencia de } \\
\text { viaje }\end{array}$ & $\begin{array}{c}\text { Frecuencia } \\
\text { absoluta }\end{array}$ & $\begin{array}{c}\text { Frecuencia } \\
\text { acumulada }\end{array}$ & $\begin{array}{c}\text { Frecuencia } \\
\text { relativa }\end{array}$ & $\begin{array}{c}\text { Frecuencia } \\
\text { relativa } \\
\text { acumulada }\end{array}$ \\
\hline 1 vez al año & 114 & 114 & $30 \%$ & $30 \%$ \\
\hline Feriados & 267 & 381 & $70 \%$ & $100 \%$ \\
\hline
\end{tabular}

El $70 \%$ de los potenciales clientes realizan viajes en época de feriados, mientras que el restante lo hace una vez en el año. 


\section{Organización de viaje}

Cuadro 9. Organización de viaje de los potenciales clientes de la ciudad de Riobamba

\begin{tabular}{|c|c|c|c|c|}
\hline Organización de viaje & $\begin{array}{c}\text { Frecuencia } \\
\text { absoluta }\end{array}$ & $\begin{array}{c}\text { Frecuencia } \\
\text { acumulada }\end{array}$ & $\begin{array}{c}\text { Frecuencia } \\
\text { relativa }\end{array}$ & $\begin{array}{c}\text { Frecuencia } \\
\text { relativa } \\
\text { acumulada }\end{array}$ \\
\hline Agencia de viajes u Operadora de turismo & 162 & 162 & $43 \%$ & $43 \%$ \\
\hline Por su propia cuenta & 219 & 381 & $58 \%$ & $100 \%$ \\
\hline
\end{tabular}

El $58 \%$ de los potenciales clientes viajan por su propia cuenta, mientras que el restante prefieren hacerlo por medio de una agencia $\mathrm{u}$ operadora de turismo.

\section{Tipo de transporte}

Cuadro 10. Tipo de transporte que utilizan los potenciales clientes de la ciudad de Riobamba cuando salen de vacaciones

\begin{tabular}{|c|c|c|c|c|}
\hline Tipo de transporte & $\begin{array}{c}\text { Frecuencia } \\
\text { absoluta }\end{array}$ & $\begin{array}{c}\text { Frecuencia } \\
\text { acumulada }\end{array}$ & $\begin{array}{c}\text { Frecuencia } \\
\text { relativa }\end{array}$ & $\begin{array}{c}\text { Frecuencia } \\
\text { relativa } \\
\text { acumulada }\end{array}$ \\
\hline Público & 210 & 210 & $55 \%$ & $55 \%$ \\
\hline Privado & 171 & 381 & $45 \%$ & $100 \%$ \\
\hline
\end{tabular}

El 55\% de los potenciales clientes utilizan transporte público al momento de salir de vacaciones y el restante usan transporte privado.

\section{Tiempo de permanencia}

Cuadro 12. Tiempo que prefieren permanecer los potenciales clientes de la ciudad de Riobamba en determinado lugar de destino

\begin{tabular}{|c|c|c|c|c|}
\hline $\begin{array}{c}\text { Tiempo de } \\
\text { permanencia }\end{array}$ & $\begin{array}{c}\text { Frecuencia } \\
\text { absoluta }\end{array}$ & $\begin{array}{c}\text { Frecuencia } \\
\text { acumulada }\end{array}$ & $\begin{array}{c}\text { Frecuencia } \\
\text { relativa }\end{array}$ & $\begin{array}{c}\text { Frecuencia } \\
\text { relativa } \\
\text { acumulada }\end{array}$ \\
\hline 1 día & 124 & 124 & $33 \%$ & $33 \%$ \\
\hline 2 días & 95 & 219 & $25 \%$ & $58 \%$ \\
\hline 3 días & 143 & 362 & $38 \%$ & $95 \%$ \\
\hline Más de 3 días & 19 & 381 & $5 \%$ & $100 \%$ \\
\hline
\end{tabular}

El 38\% de los potenciales clientes prefieren permanecer 3 días en determinado lugar, el 33\% por su lado le gustaría hacerlo por 1 día, mientras que el 25\% expresan que lo harían solo por un día.

\section{Forma de pago}

Cuadro 13. Forma de pago de los potenciales clientes de la ciudad de Riobamba

\begin{tabular}{|c|c|c|c|c|}
\hline $\begin{array}{c}\text { Forma de } \\
\text { pago }\end{array}$ & $\begin{array}{c}\text { Frecuencia } \\
\text { absoluta }\end{array}$ & $\begin{array}{c}\text { Frecuencia } \\
\text { acumulada }\end{array}$ & $\begin{array}{c}\text { Frecuencia } \\
\text { relativa }\end{array}$ & $\begin{array}{c}\text { Frecuencia relativa } \\
\text { acumulada }\end{array}$ \\
\hline Efectivo & 314 & 314 & $83 \%$ & $83 \%$ \\
\hline Crédito & 67 & 381 & $18 \%$ & $100 \%$ \\
\hline
\end{tabular}


El 82\% de los potenciales clientes prefieren realizar sus pagos en efectivo, mientras que el restante pagarían por medio de tarjeta de crédito.

\section{Ubicación de la empresa a crearse}

Cuadro 14. Ubicación de la empresa a crearse en base a la preferencia de los potenciales clientes de la ciudad de Riobamba

\begin{tabular}{|c|c|c|c|c|}
\hline Ubicación de la empresa & $\begin{array}{c}\text { Frecuencia } \\
\text { absoluta }\end{array}$ & $\begin{array}{c}\text { Frecuencia } \\
\text { acumulada }\end{array}$ & $\begin{array}{c}\text { Frecuencia } \\
\text { relativa }\end{array}$ & $\begin{array}{c}\text { Frecuencia } \\
\text { relativa } \\
\text { acumulada }\end{array}$ \\
\hline Centro histórico & 86 & 86 & $22,5 \%$ & $22,5 \%$ \\
\hline Centro moderno & 114 & 200 & $30 \%$ & $53 \%$ \\
\hline Norte de la ciudad & 181 & 381 & $47,5 \%$ & $100 \%$ \\
\hline
\end{tabular}

El 47,5\% de los potenciales clientes prefiere que la empresa a crearse se implemente en el norte de la ciudad, mientras que al 30\% le gustaría que la empresa esté ubicada en el centro moderno de Riobamba.

\section{Preferencia del turista en cuanto al nombre de la empresa}

Cuadro 15. Preferencia de los potenciales clientes de la ciudad de Riobamba en cuanto al nombre de la empresa

\begin{tabular}{|c|c|c|c|c|}
\hline $\begin{array}{c}\text { Preferencia del cliente en cuanto } \\
\text { al nombre de la empresa }\end{array}$ & $\begin{array}{c}\text { Frecuencia } \\
\text { absoluta }\end{array}$ & $\begin{array}{c}\text { Frecuencia } \\
\text { acumulada }\end{array}$ & $\begin{array}{c}\text { Frecuencia } \\
\text { relativa }\end{array}$ & $\begin{array}{c}\text { Frecuencia } \\
\text { relativa } \\
\text { acumulada }\end{array}$ \\
\hline The Sumak Tourism & 57 & 57 & $15 \%$ & $15 \%$ \\
\hline Sumak Tourism Rio & 286 & 343 & $75 \%$ & $90 \%$ \\
\hline Tour Operadora Riobamba & 38 & 381 & $10 \%$ & $100 \%$ \\
\hline
\end{tabular}

El 75\% de los potenciales clientes le gustaría que la empresa a crearse lleve como nombre "Sumak Tourism Rio", el 15\% prefiere que se llame "The Sumak Tourism” y el restante opina que debería llamarse "Tour Operadora Riobamba”.

\section{Medios que utiliza el cliente para informarse}

Cuadro 16. Medios que utiliza el potencial cliente de Riobamba para informarse

\begin{tabular}{|c|c|c|c|c|}
\hline $\begin{array}{c}\text { Medios que utiliza el cliente para } \\
\text { informarse }\end{array}$ & $\begin{array}{c}\text { Frecuencia } \\
\text { absoluta }\end{array}$ & $\begin{array}{c}\text { Frecuencia } \\
\text { acumulada }\end{array}$ & $\begin{array}{c}\text { Frecuencia } \\
\text { relativa }\end{array}$ & $\begin{array}{c}\text { Frecuencia } \\
\text { relativa } \\
\text { acumulada }\end{array}$ \\
\hline Puntos de información turística & 29 & 29 & $8 \%$ & $7,5 \%$ \\
\hline Internet & 95 & 124 & $25 \%$ & $32,5 \%$ \\
\hline Redes Sociales & 124 & 248 & $33 \%$ & $65,0 \%$ \\
\hline Amigos o familiares & 38 & 286 & $10 \%$ & $75 \%$ \\
\hline Operadora de turismo o Agencia de viajes & 38 & 324 & $10 \%$ & $85 \%$ \\
\hline Guía de viajes & 10 & 333 & $3 \%$ & $87,5 \%$ \\
\hline Radio o televisión & 48 & 381 & $13 \%$ & $100 \%$ \\
\hline
\end{tabular}


El 33\% de los potenciales clientes se informa por medio de las redes sociales, un $25 \%$ lo hace por internet, el $13 \%$ prefiere hacerlo por televisión o radio.

\section{Resumen del Perfil de los Potenciales Clientes de la Ciudad de Riobamba}

Cuadro 17. Perfil de los Potenciales Clientes de la Ciudad de Riobamba

\begin{tabular}{|c|c|c|}
\hline \multicolumn{2}{|c|}{ PERFIL DEL TURISTA } \\
\hline Edad & 25 a 29 años & $22,5 \%$ \\
\hline Género & Femenino & $65 \%$ \\
\hline Nivel de Instrucción & Superior & $42,5 \%$ \\
\hline Procedencia & Riobamba, Ambato, Guayaquil & $82,5 \%$ \\
\hline Ocupación & Empleados, estudiantes & $75 \%$ \\
\hline Tipo de Empresa a Crearse & $\begin{array}{c}\text { Operadora de turismo y agencia de } \\
\text { viajes }\end{array}$ & $70 \%$ \\
\hline Forma de Viaje & Familia & $48 \%$ \\
\hline Frecuencia de Viaje & Feriados & $70 \%$ \\
\hline Organización de Viaje & Por su propia cuenta & $58 \%$ \\
\hline Tipo de Transporte & Público & $55 \%$ \\
\hline Preferencia del Turista en cuanto a Paquetes & Paquete 4 (Nevado El Altar) & $23 \%$ \\
\hline Tiempo de permanencia & 3 días & $38 \%$ \\
\hline Forma de Pago & Efectivo & $82,5 \%$ \\
\hline Ubicación de la empresa & Norte de la ciudad & $47,5 \%$ \\
\hline Preferencia en cuanto al nombre de la empresa & Sumak Tourism Rio & $75 \%$ \\
\hline Medios que utiliza el cliente para informarse & Redes sociales, internet, radio o & $71 \%$ \\
\hline
\end{tabular}

\section{Perfil de los potenciales clientes de la ciudad de guayaquil}

\section{Edad}

Cuadro 18. Edad de los potenciales clientes de la ciudad de Guayaquil

\begin{tabular}{|c|c|c|c|c|}
\hline Edad & $\begin{array}{c}\text { Frecuencia } \\
\text { absoluta }\end{array}$ & $\begin{array}{c}\text { Frecuencia } \\
\text { acumulada }\end{array}$ & $\begin{array}{c}\text { Frecuencia } \\
\text { relativa }\end{array}$ & $\begin{array}{c}\text { Frecuencia } \\
\text { relativa } \\
\text { acumulada }\end{array}$ \\
\hline De 15 a 19 años & 31 & 31 & $8,1 \%$ & $8,1 \%$ \\
\hline De 20 a 24 años & 39 & 70 & $10,2 \%$ & $18 \%$ \\
\hline De 25 a 29 años & 156 & 226 & $40,7 \%$ & $58,9 \%$ \\
\hline De 30 a 34 años & 23 & 250 & $6,1 \%$ & $65 \%$ \\
\hline De 35 a 39 años & 34 & 284 & $8,9 \%$ & $74 \%$ \\
\hline De 40 a 44 años & 19 & 303 & $4,9 \%$ & $79 \%$ \\
\hline De 45 a 49 años & 20 & 323 & $5,3 \%$ & $84,1 \%$ \\
\hline De 50 a 54 años & 12 & 336 & $3,3 \%$ & $87 \%$ \\
\hline De 55 a 59 años & 25 & 361 & $6,5 \%$ & $94 \%$ \\
\hline De 60 a 64 años & 23 & 384 & $6,1 \%$ & $100 \%$ \\
\hline
\end{tabular}


El 40,7\% del universo de estudio tienen edades entre los 25 a 29 años, el 10,2\% tienen entre 20 y 24 años de edad y el 8,9\% se hallan entre los 35 y 39 años.

\section{Género}

Cuadro 19. Género de los potenciales clientes de la ciudad de Guayaquil

\begin{tabular}{|c|c|c|c|c|}
\hline Género & $\begin{array}{c}\text { Frecuencia } \\
\text { absoluta }\end{array}$ & $\begin{array}{c}\text { Frecuencia } \\
\text { acumulada }\end{array}$ & $\begin{array}{c}\text { Frecuencia } \\
\text { relativa }\end{array}$ & $\begin{array}{c}\text { Frecuencia relativa } \\
\text { acumulada }\end{array}$ \\
\hline Masculino & 229 & 229 & $60 \%$ & $60 \%$ \\
\hline Femenino & 155 & 384 & $40 \%$ & $100 \%$ \\
\hline
\end{tabular}

El $60 \%$ de los potenciales clientes son del género masculino y el restante del género femenino.

\section{Nivel de instrucción}

Cuadro 20. Nivel de instrucción de los potenciales clientes de la ciudad de Guayaquil

\begin{tabular}{|c|c|c|c|c|}
\hline Nivel de instrucción & $\begin{array}{c}\text { Frecuencia } \\
\text { absoluta }\end{array}$ & $\begin{array}{c}\text { Frecuencia } \\
\text { acumulada }\end{array}$ & $\begin{array}{c}\text { Frecuencia } \\
\text { relativa }\end{array}$ & $\begin{array}{c}\text { Frecuencia } \\
\text { relativa } \\
\text { acumulada }\end{array}$ \\
\hline Primaria & 5 & 5 & $1,2 \%$ & $1,2 \%$ \\
\hline Secundaria & 304 & 309 & $79,3 \%$ & $80,5 \%$ \\
\hline Superior & 73 & 382 & $19,1 \%$ & $99,6 \%$ \\
\hline Postgrado & 2 & 384 & $0,4 \%$ & $100 \%$ \\
\hline
\end{tabular}

El 79,3\% del universo de estudio tienen un nivel de instrucción secundaria, por otro lado el $19,1 \%$ han terminado o están cruzando su educación superior, mientras que el 1,2\% solo culminaron la primaria.

\section{Procedencia}

Cuadro 21. Procedencia de los potenciales clientes de la ciudad de Guayaquil

\begin{tabular}{|l|l|l|l|l|}
\hline Procedencia & $\begin{array}{l}\text { Frecuencia } \\
\text { absoluta }\end{array}$ & $\begin{array}{l}\text { Frecuencia } \\
\text { acumulada }\end{array}$ & $\begin{array}{l}\text { Frecuencia } \\
\text { relativa }\end{array}$ & $\begin{array}{l}\text { Frecuencia } \\
\text { relativa } \\
\text { acumulada }\end{array}$ \\
\hline Guayaquil & 234 & 234 & $61 \%$ & $61 \%$ \\
\hline Bucay & 47 & 281 & $12,2 \%$ & $73,2 \%$ \\
\hline Riobamba & 39 & 320 & $10,2 \%$ & $83,3 \%$ \\
\hline Ambato & 31 & 351 & $8,1 \%$ & $91,5 \%$ \\
\hline Machala & 23 & 375 & $6,1 \%$ & $97,6 \%$ \\
\hline Bucay & 9 & 384 & $2,4 \%$ & $100 \%$ \\
\hline
\end{tabular}

El 61\% de los potenciales clientes son de la ciudad de Guayaquil, el $12,2 \%$ provienen de la ciudad de Bucay, un 10,2\% proceden de Riobamba y un $8,1 \%$ vienen de Ambato. 


\section{Ocupación}

Cuadro 22. Ocupación de los potenciales clientes de la ciudad de Guayaquil

\begin{tabular}{|c|c|c|c|c|}
\hline Ocupación & $\begin{array}{c}\text { Frecuencia } \\
\text { absoluta }\end{array}$ & $\begin{array}{c}\text { Frecuencia } \\
\text { acumulada }\end{array}$ & $\begin{array}{c}\text { Frecuencia } \\
\text { relativa }\end{array}$ & $\begin{array}{c}\text { Frecuencia } \\
\text { relativa } \\
\text { acumulada }\end{array}$ \\
\hline Empleado público & 259 & 259 & $67,5 \%$ & $67,5 \%$ \\
\hline Empleado privado & 45 & 304 & $11,8 \%$ & $79,3 \%$ \\
\hline Estudiante & 42 & 347 & $11 \%$ & $90,2 \%$ \\
\hline Jubilado & 17 & 364 & $4,5 \%$ & $94,7 \%$ \\
\hline Ama de casa & 20 & 384 & $5,3 \%$ & $100 \%$ \\
\hline
\end{tabular}

El 67,5\% del universo de estudio son empleados del sector público, por otro lado el 11,8\% laboran en el sector privado, mientras que el 11\% son estudiantes.

\section{Tipo de empresa a crearse}

Cuadro 23. Tipo de empresa a crearse según la preferencia de la población de Guayaquil

\begin{tabular}{|c|c|c|c|c|}
\hline Tipo de empresa a crearse & $\begin{array}{c}\text { Frecuencia } \\
\text { absoluta }\end{array}$ & $\begin{array}{c}\text { Frecuencia } \\
\text { acumulada }\end{array}$ & $\begin{array}{c}\text { Frecuencia } \\
\text { relativa }\end{array}$ & $\begin{array}{c}\text { Frecuencia } \\
\text { relativa } \\
\text { acumulada }\end{array}$ \\
\hline $\begin{array}{c}\text { Operadora de turismo y } \\
\text { agencia de viajes }\end{array}$ & 272 & 272 & $71 \%$ & $71 \%$ \\
\hline Establecimiento hotelero & 84 & 356 & $22 \%$ & $93 \%$ \\
\hline $\begin{array}{c}\text { Establecimiento de comidas } \\
\text { y bebidas }\end{array}$ & 28 & 384 & $7 \%$ & $100 \%$ \\
\hline
\end{tabular}

El 71,4\% de los potenciales clientes prefieren que se implemente una operadora de turismo y agencia de viajes, el 22\% le gustaría que se cree un establecimiento hotelero y el restante está de acuerdo que se establezca un establecimiento de comidas y bebidas.

\section{Forma de viaje}

Cuadro 24. Forma de viajar de los potenciales clientes de la ciudad de Guayaquil

\begin{tabular}{|c|c|c|c|c|}
\hline Forma de viaje & $\begin{array}{c}\text { Frecuencia } \\
\text { absoluta }\end{array}$ & $\begin{array}{c}\text { Frecuencia } \\
\text { acumulada }\end{array}$ & $\begin{array}{c}\text { Frecuencia } \\
\text { relativa }\end{array}$ & $\begin{array}{c}\text { Frecuencia } \\
\text { relativa } \\
\text { acumulada }\end{array}$ \\
\hline Sólo & 17 & 17 & $4 \%$ & $4 \%$ \\
\hline Pareja & 80 & 97 & $21 \%$ & $25 \%$ \\
\hline Familia & 189 & 286 & $49 \%$ & $74 \%$ \\
\hline Amigos & 83 & 368 & $22 \%$ & $96 \%$ \\
\hline Compañeros & 16 & 384 & $4 \%$ & $100 \%$ \\
\hline
\end{tabular}

El 49\% de los potenciales clientes prefieren viajar en familia, el 22\% viajan con amigos, y por su parte el $21 \%$ viajan con su pareja. 


\section{Frecuencia de viaje}

Cuadro 25. Frecuencia con la que los potenciales clientes de la ciudad de Guayaquil viajan

\begin{tabular}{|c|c|c|c|c|}
\hline $\begin{array}{c}\text { Frecuencia de } \\
\text { viaje }\end{array}$ & $\begin{array}{c}\text { Frecuencia } \\
\text { absoluta }\end{array}$ & $\begin{array}{c}\text { Frecuencia } \\
\text { acumulada }\end{array}$ & $\begin{array}{c}\text { Frecuencia } \\
\text { relativa }\end{array}$ & $\begin{array}{c}\text { Frecuencia } \\
\text { relativa } \\
\text { acumulada }\end{array}$ \\
\hline 1 vez al año & 318 & 318 & $83 \%$ & $83 \%$ \\
\hline Feriados & 66 & 384 & $17 \%$ & $100 \%$ \\
\hline
\end{tabular}

El 83\% de los potenciales clientes realizan viajes una vez al año, mientras que el restante lo hace en época de feriados.

\section{Organización de viaje}

Cuadro 26. Organización de viaje de los potenciales clientes de la ciudad de Guayaquil

\begin{tabular}{|c|c|c|c|c|}
\hline Organización de viaje & $\begin{array}{c}\text { Frecuencia } \\
\text { absoluta }\end{array}$ & $\begin{array}{c}\text { Frecuencia } \\
\text { acumulada }\end{array}$ & $\begin{array}{c}\text { Frecuencia } \\
\text { relativa }\end{array}$ & $\begin{array}{c}\text { Frecuencia } \\
\text { relativa } \\
\text { acumulada }\end{array}$ \\
\hline Agencia de viajes u Operadora de turismo & 89 & 89 & $23 \%$ & $23 \%$ \\
\hline Por su propia cuenta & 295 & 384 & $77 \%$ & $100 \%$ \\
\hline
\end{tabular}

El $77 \%$ de los potenciales clientes viajan por su propia cuenta, mientras que el restante prefieren hacerlo por medio de una agencia $u$ operadora de turismo.

\section{Tipo de transporte}

Cuadro 27. Tipo de transporte que utilizan los potenciales clientes de la ciudad de Guayaquil cuando salen de vacaciones

\begin{tabular}{|c|c|c|c|c|}
\hline Tipo de transporte & $\begin{array}{c}\text { Frecuencia } \\
\text { absoluta }\end{array}$ & $\begin{array}{c}\text { Frecuencia } \\
\text { acumulada }\end{array}$ & $\begin{array}{c}\text { Frecuencia } \\
\text { relativa }\end{array}$ & $\begin{array}{c}\text { Frecuencia } \\
\text { relativa } \\
\text { acumulada }\end{array}$ \\
\hline Público & 167 & 167 & $43 \%$ & $43 \%$ \\
\hline Privado & 217 & 384 & $57 \%$ & $100 \%$ \\
\hline
\end{tabular}

El $57 \%$ de los potenciales clientes utilizan transporte privado al momento de salir de vacaciones y el restante usa transporte público.

\section{Preferencia del turista en cuanto a paquetes turísticos}

Cuadro 28. Preferencia del cliente de Guayaquil en cuanto a paquetes turísticos

\begin{tabular}{|c|c|c|c|c|}
\hline $\begin{array}{c}\text { Preferencia en cuanto a paquetes } \\
\text { turísticos }\end{array}$ & $\begin{array}{c}\text { Frecuencia } \\
\text { absoluta }\end{array}$ & $\begin{array}{c}\text { Frecuencia } \\
\text { acumulada }\end{array}$ & $\begin{array}{c}\text { Frecuencia } \\
\text { relativa }\end{array}$ & $\begin{array}{c}\text { Frecuencia } \\
\text { relativa } \\
\text { acumulada }\end{array}$ \\
\hline Paquete 1 (Nevado Chimborazo) & 77 & 77 & $20 \%$ & $20 \%$ \\
\hline Paquete 2 (Lagunas de Atillo) & 58 & 134 & $15 \%$ & $35 \%$ \\
\hline Paquete 3 (Camino del Inca) & 48 & 182 & $12,5 \%$ & $47,5 \%$ \\
\hline Paquete 4 (Nevado el Altar) & 58 & 240 & $15 \%$ & $62,5 \%$ \\
\hline Paquete 5 (México) & 29 & 269 & $7,5 \%$ & $70 \%$ \\
\hline Paquete 6 (Perú) & 19 & 288 & $5 \%$ & $75 \%$ \\
\hline Paquete 7 (EE.UU) & 96 & 384 & $25 \%$ & $100 \%$ \\
\hline
\end{tabular}


El 23\% de los potenciales clientes de la ciudad de Riobamba le gustaría adquirir un paquete para conocer el nevado El Altar, por otro lado el $20 \%$ preferirían viajar a las Lagunas de Atillo, mientras que el 18\% optarían por el paquete turístico del Camino del Inca y un 13\% escogieron el paquete turístico hacia el Perú valga la redundancia.

\section{Tiempo de permanencia}

Cuadro 29. Tiempo que prefieren permanecer los potenciales clientes de la ciudad de Guayaquil en determinado lugar de destino

\begin{tabular}{|c|c|c|c|c|}
\hline $\begin{array}{c}\text { Tiempo de } \\
\text { permanencia }\end{array}$ & Frecuencia absoluta & $\begin{array}{c}\text { Frecuencia } \\
\text { acumulada }\end{array}$ & $\begin{array}{c}\text { Frecuencia } \\
\text { relativa }\end{array}$ & $\begin{array}{c}\text { Frecuencia } \\
\text { relativa } \\
\text { acumulada }\end{array}$ \\
\hline 1 día & 8 & 8 & $2 \%$ & $2 \%$ \\
\hline 2 días & 80 & 87 & $21 \%$ & $23 \%$ \\
\hline 3 días & 253 & 340 & $66 \%$ & $89 \%$ \\
\hline Más de 3 días & 44 & 384 & $11 \%$ & $100 \%$ \\
\hline
\end{tabular}

El 66\% de los potenciales clientes prefieren permanecer 3 días en determinado lugar, el $21 \%$ por su lado le gustaría hacerlo por 2 días, mientras que el 11\% expresan que lo harían por más de 3 días.

\section{Forma de pago}

Cuadro 30. Forma de pago de los potenciales clientes de la ciudad de Guayaquil

\begin{tabular}{|l|l|l|l|ll|}
\hline $\begin{array}{l}\text { Forma de } \\
\text { pago }\end{array}$ & $\begin{array}{l}\text { Frecuencia } \\
\text { absoluta }\end{array}$ & $\begin{array}{l}\text { Frecuencia } \\
\text { acumulada }\end{array}$ & $\begin{array}{l}\text { Frecuencia } \\
\text { relativa }\end{array}$ & $\begin{array}{l}\text { Frecuencia relativa } \\
\text { acumulada }\end{array}$ & \\
\hline Efectivo & 379 & 379 & $99 \%$ & $99 \%$ \\
\hline Crédito & 5 & 384 & $1 \%$ & $100 \%$ & \\
\hline
\end{tabular}

El 99\% de los potenciales clientes prefieren realizar sus pagos en efectivo, mientras que el restante pagarían por medio de tarjeta de crédito.

\section{Ubicación de la empresa a crearse}

Cuadro 31. Ubicación de la empresa a crearse en base a la preferencia de los potenciales clientes de la ciudad de Guayaquil

\begin{tabular}{|c|c|c|c|c|}
\hline Ubicación de la empresa & $\begin{array}{c}\text { Frecuencia } \\
\text { absoluta }\end{array}$ & $\begin{array}{c}\text { Frecuencia } \\
\text { acumulada }\end{array}$ & $\begin{array}{c}\text { Frecuencia } \\
\text { relativa }\end{array}$ & $\begin{array}{c}\text { Frecuencia } \\
\text { relativa } \\
\text { acumulada }\end{array}$ \\
\hline Centro histórico & 125 & 125 & $32,5 \%$ & $32,5 \%$ \\
\hline Centro moderno & 25 & 150 & $6,5 \%$ & $39 \%$ \\
\hline Norte de la ciudad & 234 & 384 & $61 \%$ & $100 \%$ \\
\hline
\end{tabular}

El 61\% de los potenciales clientes prefiere que la empresa a crearse se implemente en el norte de la ciudad, mientras que al 32,5\% le gustaría que la empresa esté ubicada en el centro histórico de Riobamba. 


\section{Preferencia del turista en cuanto al nombre de la empresa}

Cuadro 32. Preferencia de los potenciales clientes de la ciudad de Guayaquil en cuanto al nombre de la empresa

\begin{tabular}{|c|c|c|c|c|}
\hline $\begin{array}{c}\text { Preferencia del cliente en } \\
\text { cuanto al nombre de la } \\
\text { empresa }\end{array}$ & $\begin{array}{c}\text { Frecuencia } \\
\text { absoluta }\end{array}$ & $\begin{array}{c}\text { Frecuencia } \\
\text { acumulada }\end{array}$ & $\begin{array}{c}\text { Frecuencia } \\
\text { relativa }\end{array}$ & $\begin{array}{c}\text { Frecuencia } \\
\text { relativa } \\
\text { acumulada }\end{array}$ \\
\hline The Sumak Tourism & 109 & 109 & $28 \%$ & $28 \%$ \\
\hline Sumak Tourism Rio & 203 & 312 & $53 \%$ & $81 \%$ \\
\hline Tour Operadora Riobamba & 72 & 384 & $19 \%$ & $100 \%$ \\
\hline
\end{tabular}

El 53\% de los potenciales clientes le gustaría que la empresa a crearse lleve como nombre "Sumak Tourism Rio", el 28\% prefiere que se llame “The Sumak Tourism” y el restante opina que debería llamarse "Tour Operadora Riobamba”.

\section{Medios que utiliza el cliente para informarse}

Cuadro 33. Medios que utiliza el potencial cliente de Guayaquil para informarse

\begin{tabular}{|c|c|c|c|c|}
\hline $\begin{array}{c}\text { Medios que utiliza el cliente para } \\
\text { informarse }\end{array}$ & $\begin{array}{c}\text { Frecuencia } \\
\text { absoluta }\end{array}$ & $\begin{array}{c}\text { Frecuencia } \\
\text { acumulada }\end{array}$ & $\begin{array}{c}\text { Frecuencia } \\
\text { relativa }\end{array}$ & $\begin{array}{c}\text { Frecuencia } \\
\text { relativa } \\
\text { acumulada }\end{array}$ \\
\hline Puntos de información turística & 28 & 28 & $7 \%$ & $7,3 \%$ \\
\hline Internet & 109 & 137 & $28 \%$ & $35,8 \%$ \\
\hline Redes Sociales & 156 & 293 & $41 \%$ & $76,4 \%$ \\
\hline Omigos o familiares & 17 & 311 & $4 \%$ & $81 \%$ \\
\hline Operadora de turismo o Agencia de viajes & 8 & 318 & $2 \%$ & $83 \%$ \\
\hline Radio o televisión & 19 & 337 & $5 \%$ & $87,8 \%$ \\
\hline
\end{tabular}

El 41\% de los potenciales clientes se informa por medio de las redes sociales, un $28 \%$ lo hace por internet, el $12 \%$ prefiere hacerlo por televisión o radio.

\section{Resumen del Perfil de los Potenciales Clientes de la Ciudad de Guayaquil}

Cuadro 34. Perfil de los Potenciales Clientes de la Ciudad de Guayaquil

\begin{tabular}{|c|c|c|}
\hline \multicolumn{2}{|c|}{ PERFIL DEL TURISTA } \\
\hline Edad & 25 a 29 años & $40,7 \%$ \\
\hline Género & Masculino & $60 \%$ \\
\hline Nivel de Instrucción & Secundaria & $79,3 \%$ \\
\hline Procedencia & Guayaquil, Bucay, Riobamba & $83,3 \%$ \\
\hline Ocupación & Empleados, estudiantes & $90,2 \%$ \\
\hline Tipo de Empresa a Crearse & Operadora de turismo y agencia de & $71 \%$ \\
\hline Forma de Viaje & viajes & $49 \%$ \\
\hline Frecuencia de Viaje & Familia & $83 \%$ \\
\hline Organización de Viaje & 1 vez al año & $77 \%$ \\
\hline Tipo de Transporte & Por su propia cuenta & $57 \%$ \\
\hline
\end{tabular}




\begin{tabular}{|c|c|c|}
\hline $\begin{array}{c}\text { Preferencia del Turista en cuanto a Paquetes } \\
\text { Turísticos }\end{array}$ & Paquete 7 (Estados Unidos) & $25 \%$ \\
\hline Tiempo de permanencia & 3 días & $66 \%$ \\
\hline Forma de Pago & Efectivo & $99 \%$ \\
\hline Ubicación de la empresa & Norte de la ciudad & $61 \%$ \\
\hline Preferencia en cuanto al nombre de la empresa & Sumak Tourism Rio & $53 \%$ \\
\hline Medios que utiliza el cliente para informarse & $\begin{array}{c}\text { Redes sociales, internet, radio o } \\
\text { televisión }\end{array}$ & $81 \%$ \\
\hline
\end{tabular}

\section{Análisis de la competencia}

Se consideró como competencia a las empresas turísticas (Agencias de viajes y operadoras de turismo) de la ciudad de Riobamba que ofrecen productos turísticos de similares características que se presenta en el cuadro a continuación:

Cuadro 35. Competencia actual

\begin{tabular}{|c|c|}
\hline Denominación. & Clientes. \\
\hline DELGADO TRAVEL & 115 \\
\hline MAJESTOURING & 126 \\
\hline RIO TUR TRAVEL & 134 \\
\hline PROBICI & 136 \\
\hline JULIO VERNE & 144 \\
\hline VELOZ CORONADO EXPEDICIONES & 139 \\
\hline TURISTARAPUYA & 137 \\
\hline UNIVERSAL CENTER TRAVEL & 139 \\
\hline CHIMBORAZO BEAUTY & 140 \\
\hline CIRCULO TOURS & 155 \\
\hline RUMBO TRAVEL & 153 \\
\hline INCAÑAN ECO TOURISM S.A. & 158 \\
\hline SUSTAIN TOURS & 142 \\
\hline PURUHA RAZURKU & 144 \\
\hline SOUL TRAIN & 145 \\
\hline ANDESSPIRIT & 147 \\
\hline MUNDODESTINOS & 149 \\
\hline CACHA ALLISAMAY & 150 \\
\hline ALTAR CLIMBING & 152 \\
\hline SALAZARTOURS & 153 \\
\hline ANDEAN ADVENTURES & 155 \\
\hline ATRIPEAR & 156 \\
\hline ECUAKAWSANI & 126 \\
\hline VIDA AVENTURERA ORNA TRAVEL S.A. & 128 \\
\hline EXPEDICIONES ANDINAS & 130 \\
\hline MUNDO TOURS & 133 \\
\hline GUI\&EME & 134 \\
\hline AEROVACACIONES & 137 \\
\hline INFINITYECUADOR & 139 \\
\hline AGLOTOUR AGENCIA DE VIAJES GLOBAL TOUR CIA. LTDA. & 141 \\
\hline COMPETENCIA ACTUAL: & 4238 \\
\hline
\end{tabular}


En dicho cuadro se puede evidenciar las principales empresas turísticas (Agencias de Viajes y Operadoras de Turismo) que funcionan en la ciudad de Riobamba y ofertan productos turísticos de similares características con la diferencia que se centran en ofrecerlos a turistas extranjeros.

Proyección de la Demanda y Competencia Futura Demanda futura del universo de la ciudad de Riobamba La demanda futura se calculó en base a la fórmula de incremento compuesto cuyo modelo matemático es:

$$
\text { Cn= Co (1+i) }{ }^{n}
$$

\begin{tabular}{|c|c|}
\hline $\mathrm{Co}=$ & 51631 \\
\hline $\mathrm{i}=$ & $1.5 \%$ \\
\hline
\end{tabular}

Según los datos del censo de población y vivienda del año 2010, la tasa de crecimiento poblacional de la provincia de Chimborazo es del $\mathbf{1 , 5 \%}$ cada año. (INEC 2010).

Cuadro 36. Demanda futura del universo de la ciudad de Riobamba

\begin{tabular}{|c|c|}
\hline Año & Clientes \\
\hline 2015 & 51631 \\
\hline 2016 & 52405 \\
\hline 2017 & 53192 \\
\hline 2018 & 53989 \\
\hline 2019 & 54799 \\
\hline 2020 & 55621 \\
\hline 2021 & 56456 \\
\hline 2022 & 57302 \\
\hline 2023 & 58162 \\
\hline 2024 & 59034 \\
\hline 2025 & 59920 \\
\hline
\end{tabular}

Para proyectar la demanda futura para los próximos 10 años de turismo interno se tomó como base la tasa de crecimiento poblacional de la provincia de Chimborazo. 
Demanda futura del universo de la ciudad de Guayaquil

La demanda futura se calculó en base a la fórmula de incremento compuesto cuyo modelo matemático es:

$$
\operatorname{Cn}=\operatorname{Co}(1+\mathbf{i})^{n}
$$

\begin{tabular}{|c|c|}
\hline $\mathrm{Co}=$ & 553497 \\
\hline $\mathrm{i}=$ & $1.08 \%$ \\
\hline
\end{tabular}

Según los datos del censo de población y vivienda del año 2010, la tasa de crecimiento poblacional de la provincia de Guayas es del 1,08\% cada año. (INEC 2010).

Cuadro 37. Demanda futura del universo de la ciudad de Guayaquil

\begin{tabular}{|c|c|}
\hline Año & Clientes \\
\hline 2015 & 553497 \\
\hline 2016 & 559475 \\
\hline 2017 & 565517 \\
\hline 2018 & 571625 \\
\hline 2019 & 577798 \\
\hline 2020 & 584038 \\
\hline 2021 & 590346 \\
\hline 2023 & 596722 \\
\hline 2024 & 603166 \\
\hline 2025 & 609681 \\
\hline
\end{tabular}

Para proyectar la demanda futura para los próximos 10 años de turismo interno se tomó como base la tasa de crecimiento poblacional de la provincia del Guayas.

\section{Competencia futura}

La competencia futura se calculó en base a la fórmula de incremento compuesto cuyo modelo matemático es:

$$
\text { Cn= Co (1+i) }{ }^{n}
$$

\begin{tabular}{|c|c|}
\hline $\mathrm{Co}=$ & 4238 \\
\hline $\mathrm{i}=$ & $1,24 \%$ \\
\hline
\end{tabular}


Según los datos del censo de población y vivienda del año 2010, la tasa de crecimiento poblacional del país es del 1,24\% cada año. (INEC).

Cuadro 38. Competencia futura

\begin{tabular}{|c|c|}
\hline Año & Clientes \\
\hline 2015 & 4238 \\
\hline 2016 & 4291 \\
\hline 2017 & 4344 \\
\hline 2018 & 4398 \\
\hline 2019 & 4452 \\
\hline 2020 & 4507 \\
\hline 2021 & 4563 \\
\hline 2022 & 4620 \\
\hline 2023 & 4677 \\
\hline 2024 & 4735 \\
\hline 2025 & 4794 \\
\hline
\end{tabular}

Para proyectar la competencia futura para los próximos 10 años de turismo interno se tomó como base la tasa de crecimiento poblacional del país.

Confrontación de la demanda vs oferta

Demanda insatisfecha de la ciudad de Riobamba

\begin{tabular}{|c|c|c|c|}
\multicolumn{4}{|c}{ Cuadro 39. Demanda insatisfecha de la ciudad de Riobamba } \\
\hline Año & Demanda futura & Competencia futura & Demanda insatisfecha \\
\hline 2016 & 52405 & 4291 & 48115 \\
\hline 2017 & 53192 & 4344 & 48848 \\
\hline 2018 & 53989 & 4398 & 49592 \\
\hline 2019 & 54799 & 4452 & 50347 \\
\hline 2020 & 55621 & 4507 & 51114 \\
\hline 2021 & 56456 & 4563 & 51892 \\
\hline 2022 & 57302 & 4620 & 52683 \\
\hline 2023 & 58162 & 4677 & 53485 \\
\hline 2024 & 59034 & 4735 & 54299 \\
\hline 2025 & 59920 & 4794 & 55126 \\
\hline
\end{tabular}


Demanda insatisfecha de la ciudad de Guayaquil

Cuadro 40. Demanda insatisfecha de la ciudad de Guayaquil

\begin{tabular}{|c|c|c|c|}
\hline Año & Demanda futura & Competencia futura & Demanda insatisfecha \\
\hline 2016 & 559475 & 4291 & 555184 \\
\hline 2017 & 565517 & 4344 & 561173 \\
\hline 2018 & 571625 & 4398 & 567227 \\
\hline 2019 & 577798 & 4452 & 573346 \\
\hline 2020 & 584038 & 4507 & 579531 \\
\hline 2021 & 590346 & 4563 & 585783 \\
\hline 2022 & 596722 & 4620 & 592102 \\
\hline 2023 & 603166 & 4677 & 598489 \\
\hline 2024 & 609681 & 4735 & 604945 \\
\hline 2025 & 616265 & 4794 & 611471 \\
\hline
\end{tabular}

\section{Conclusión}

Los potenciales clientes muestran diferentes intereses en cuanto a paquetes turísticos que ofrece la empresa lo que permitirá obtener beneficios económicos y de esa forma dinamizar la economía de la ciudad y por consiguiente del país, en sí mejorar la calidad de vida de la población de forma directa e indirecta.

El proyecto es ambientalmente viable, debido a que las acciones positivas tienen un porcentaje mayor, esto se debe a que la actividad turística en nuestro proyecto está totalmente basada en la sostenibilidad para todos los factores que influyen en dicha actividad.

La comercialización de los paquetes turísticos permitirá la capitalización de la empresa generando una rentabilidad positiva, pues la inversión realizada se recuperará en el primer año de operación proporcionando una tasa interna de retorno del 1493\%, un valor actual neto de \$ \$2.625.610,27 dólares y una relación beneficio costo de \$104,19 dólares.

\section{References:}

Barómetro Turístico 2011

INEC (Instituto Nacional de Estadísticas y Censos)

MCPEYC. "Ministerio Coordinador de Producción, Empleo Y Competitividad”. 2014

MINTUR. “Ministerio de Turismo del Ecuador”. 2013

OMT, (Organización Mundial de Turismo). "Guía para administradores locales: Desarrollo turístico Sostenible”. Madrid - España: Editorial Folio SENPLADES. "Secretaría Nacional de Planificación y Desarrollo - Plan Nacional de Desarrollo / Plan Nacional para el Buen Vivir”. 2013-2017 Resolución 2 Registro Oficial Suplemento 78 de 11-sep-2013 Estado. (20132017) Pp. 13 


\section{Anexos}

Anexo 1. Encuesta

ENCUESTA

ESCUELA SUPERIOR POLITÉCNICA DE CHIMBORAZO

FACULTAD DE RECURSOS NATURALES

ESCUELA DE INGENIERÍA EN ECOTURISMO

\section{ENCUESTA}

Estimado señor o señorita, esperamos su colaboración respondiendo sinceramente las siguientes preguntas, los datos por usted proporcionados serán de gran ayuda para la elaboración del proyecto. La información es confidencial y no lo compromete en nada.
a. EdadDe 15 a 19 años
b. De 20 a 24 años
c. De 25 a 29 años
d. De 30 a 34 años
e. De 35 a 39 años
f. De 40 a 44 años
g. De 45 a 49 años
h. De 50 a 54 años
i. De 55 a 59 años
j. De 60 a 64 años
a. GéneroMasculino
b. Femenino
1. Procedencia
a. Nivel de InstrucciónPrimaria
b. Secundaria
c. Superior
a. PostgradoOcupaciónEmpleado público
b. Empleado privado
c. Estudiante
d. Jubilado

2. Ama de casa ¿Qué tipo de empresa turística le gustaría que se implemente en la ciudad de Riobamba?

a. Agencia de viajes y Operadora de turismo

b. Establecimiento hotelero

c. Establecimiento de comidas y bebidas

3. Forma de viaje

a. Sólo

b. Pareja

c. Familia

d. Amigos

e. Compañeros

4. ¿Con que frecuencia sale de vacaciones?

a. 1 vez al año

b. Feriados

5. ¿Quién organiza su viaje?

a. Operadora o Agencia 
b. Por su propia cuenta

6. Tipo de transporte que utiliza para salir de vacaciones

a. Público

b. Privado

7. Preferencia de paquetes turísticos (Escoja una opción)

a. $\quad$ Paquete 1 (Nevado Chimborazo)

b. $\quad$ Paquete 2 (Lagunas de Atillo)

c. $\quad$ Paquete 3 (Camino del Inca)

d. Paquete 4 (Nevado el Altar)

e. $\quad$ Paquete 5 (México)

f. Paquete 6 (Perú)

g. $\quad$ Paquete 7 (EE.UU)

8. ¿Cuánto tiempo le gustaría permanecer en el lugar destino?

a. 1 día

b. 2 días

c. 3 días

d. Más de 3 días

9. ¿Cuál sería su forma de pago?

a. Efectivo

b. Crédito

10. Ubicación del negocio

a. Centro histórico

b. Centro moderno

c. Norte de la ciudad

11. Nombre de la empresa (Escoja una opción)

a. The Sumak Tourism

b. Sumak Tourism Rio

12. Tour Operadora RiobambaQué medios utiliza para informarse del lugar de destino

a. Puntos de información turística

b. Internet

c. Redes Sociales

d. Amigos o familiares

e. Operadora de turismo o Agencia de viajes

f. Guía de viajes

g. Radio o televisión 
Anexo 2. Catastro turístico- Agencias de viajes y operadoras de turismo

\begin{tabular}{|c|c|c|c|}
\hline NOMBRE DE LA EMPRESA & ACTIVIDAD & SUBACTIVIDAD & CATEGORIA \\
\hline CHIMBORAZO TRAVEL & Agencias de viajes & Agencias de viajes & Internacional \\
\hline DIAMANTE TOURS & Agencias de viajes & Agencias de viajes & Dualidad \\
\hline MAJESTOURING & Agencias de viajes & Agencias de viajes & Internacional \\
\hline RIO TUR TRAVEL & Agencias de viajes & Agencias de viajes & Internacional \\
\hline PROBICI & Agencias de viajes & Agencias de viajes & Operadora \\
\hline JULIO VERNE & Agencias de viajes & Agencias de viajes & Operadora \\
\hline VELOZ CORONADO EXPEDICIONES & Agencias de viajes & Agencias de viajes & Operadora \\
\hline TURISTARAPUYA & Agencias de viajes & Agencias de viajes & Operadora \\
\hline UNIVERSAL CENTER TRAVEL & Agencias de viajes & Agencias de viajes & Dualidad \\
\hline CHIMBORAZO BEAUTY & Agencias de viajes & Agencias de viajes & Operadora \\
\hline CIRCULO TOURS & Agencias de viajes & Agencias de viajes & Operadora \\
\hline RUMBO TRAVEL & Agencias de viajes & Agencias de viajes & Operadora \\
\hline INCAÑAN ECO TOURISM S.A. & Agencias de viajes & Agencias de viajes & Dualidad \\
\hline SUSTAIN TOURS & Agencias de viajes & Agencias de viajes & Dualidad \\
\hline PURUHA RAZURKU & Agencias de viajes & Agencias de viajes & Dualidad \\
\hline SOUL TRAIN & Agencias de viajes & Agencias de viajes & Operadora \\
\hline ANDESSPIRIT & Agencias de viajes & Agencias de viajes & Operadora \\
\hline MUNDODESTINOS & Agencias de viajes & Agencias de viajes & Internacional \\
\hline CACHA ALLISAMAY & Agencias de viajes & Agencias de viajes & Operadora \\
\hline ALTAR CLIMBING & Agencias de viajes & Agencias de viajes & Operadora \\
\hline SALAZARTOURS & Agencias de viajes & Agencias de viajes & Operadora \\
\hline ANDEAN ADVENTURES & Agencias de viajes & Agencias de viajes & Operadora \\
\hline ATRIPEAR & Agencias de viajes & Agencias de viajes & Operadora \\
\hline ECUAKAWSANI & Agencias de viajes & Agencias de viajes & Dualidad \\
\hline EXPEDICIONES ANDINAS & Agencias de viajes & Agencias de viajes & Operadora \\
\hline MUNDO TOURS & Agencias de viajes & Agencias de viajes & Internacional \\
\hline GUI\&EME & Agencias de viajes & Agencias de viajes & Operadora \\
\hline AEROVACACIONES & Agencias de viajes & Agencias de viajes & Dualidad \\
\hline INFINITYECUADOR & Agencias de viajes & Agencias de viajes & Dualidad \\
\hline
\end{tabular}

